\title{
The Computer-aided Design of the Multiplexer
}

\author{
Meng Pan \\ Communication University of China \\ Beijing,China,18810855972 \\ e-mail:come907@sina.com
}

\author{
HongJuan Tang \\ Communication University of China \\ Beijing,China,18810532149 \\ e-mail: xiaoerlang8855@163.com
}

\begin{abstract}
The difficult of multiplexer design is to eliminate residual susceptance. In order to eliminate residual susceptance obtaining impedance matching ,this paper designs the computeraided design software of star-point diplexer, star-point triplexer and constant impedance diplexer based on the Matlab development platform. The software can emulate the scattering matrix of the multiplexer accurately, in accordance with the SNP documents. It can also optimize the length of the transmission line rapidly achieving the purpose of eliminating residual susceptance and reducing the multiplexer design cycle. Finally, this paper verifies the correctness of the software using ADS, it is more intelligent, efficient, convenient compared with ADS.
\end{abstract}

Keywords-Multiplexer; Matlab ; Complex interpolation;Scattering parameters;ADS

\section{INTRODUCTION}

With the development of communication technology, the multiplexer become an indispensable part in the field of radio, television, microwave communication technology and so on. Multiplexer combine several frequency bands for transmission via a common antenna. In the field of radio and television, it generally has several common types of multiplexer: the starpoint multiplexer, constant impedance multiplexer and stretchline multiplexer. This paper has designed a computer-aided design software, which can calculate $\mathrm{S}$ parameters of the multiplexer according to the SNP files. It can also adjust the length of the transmission lines connecting the various composite devices, optimize the multiplexer rapidly. It is more intelligent, efficient, convenient compared with ADS. Figure 1 shows the computer-aided design software.

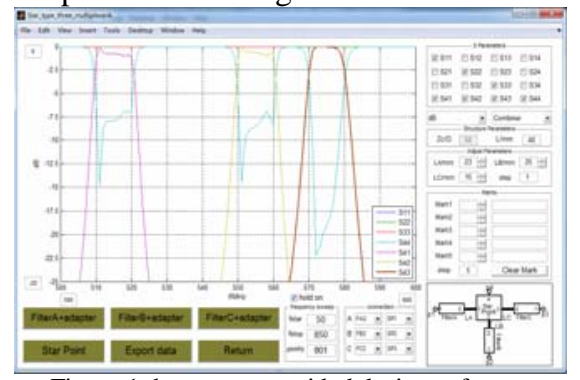

Figure 1 the computer-aided design software

\section{PRINCIPLE OF MULTIPLEXER}

\section{A. Star-point multiplexer}

Star-point multiplexer is consisted by filters and branch connectors. Figure 2 shows the star-point diplexer. At port p1,signal F1 transmits to the branch connector through a band pass filter A and the transmission of the transmission line LA, at port p1,signal F2 transmits to the branch connector through a band pass filter $\mathrm{B}$ and the transmission of the transmission line LB. In order to guarantee the isolation between the signal, the impedance from the branch connector to the left on the F2 signals should be open , and the impedance from the branch connector to the right on the F1 signals should be open, which can be achieved by adjusting the length of the LA and LB. when increasing the number of channels connected to the branch connector for the multiplexer, we should optimize the length of the transmission line[1]. For example, there are three channels connected to the branch connector. For any one channel, the impedance form branch connector to the transmission line connecting the remaining two channels should be open. Generally, it is difficult to eliminate residual susceptance achieving the target. The computer-aided design software is very good to solve this problem.

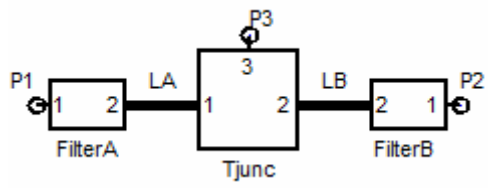

Figure 2 Star-point diplexer

\section{B. Constant impedance multiplexer}

Constant impedance multiplexer is consisted by $3 \mathrm{~dB}$ coupler, band pass filter and the absorbing load. Figure 3 shows the schematic of the constant impedance diplexer. The low frequency signal F1 inputting from $\mathrm{p} 1$ port was divided into two with the same power by $3 \mathrm{~dB}$ coupler, then through the band pass filter unimpeded, transmitting to the next $3 \mathrm{~dB}$ coupler. At port p3, the signals have the same phase, superimposing and transmitting via the antenna. At port $\mathrm{p} 2$, the signals cancel each other out because of the opposite phase.

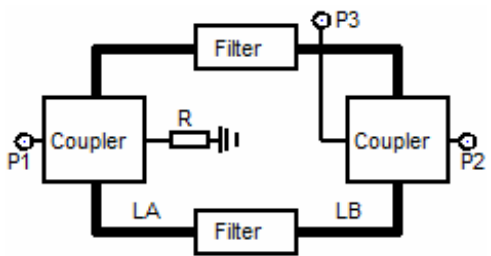

Figure 3 constant impedance diplexer

The signal F1 will not enter the port p2, causing crosstalk on the signal F2. The high frequency signal F2 inputting from p2 port is also divided into two with the same power by $3 \mathrm{~dB}$ coupler. The signals are reflected by band-pass filters 
completely and transmit to $3 \mathrm{~dB}$ coupler in the left again, superimposing at $\mathrm{p} 3$ port and offsetting at p2 port.

\section{CALCUATION OF PARAMETER}

\section{A. Influence on Reference surface mobile scattering parameter}

The scattering parameter of filters is usually obtained by measurement and the scattering parameter after the abstraction of filters should be reckoned. The algorithm and devices here are the same as the ones after the connection of transmission lines, so they can all be regarded as the movement of the network reference surface. For N-port network, let the mobile distance of each port reference surface be $d_{1}, d_{2}, \cdots d_{n}$ and the corresponding electrical length $\theta_{1}, \theta_{2}, \cdots, \theta_{n}$. Let $\theta$ be plus when the reference surface moves outward and minus when the reference surface moves inward. Let diagonal matrix $d$ be

$$
d=\operatorname{diag}\left[e^{-j \theta_{1}}, e^{-j \theta_{2}}, \cdots, e^{-j \theta_{n}}\right]
$$

Then the scattering parameter after the movement of the reference surface is [3]

$$
S^{\prime}=d S d
$$

\section{B. The algorithm to obtain the scattering parameter after connection}

We assume that one complicated microwave system consists of $\mathrm{p}$ branch networks which have $\mathrm{n}$ ports including $\mathrm{m}$ unconnected ports and n-m connected ports. Figure 4 gives $n$ port network connection.

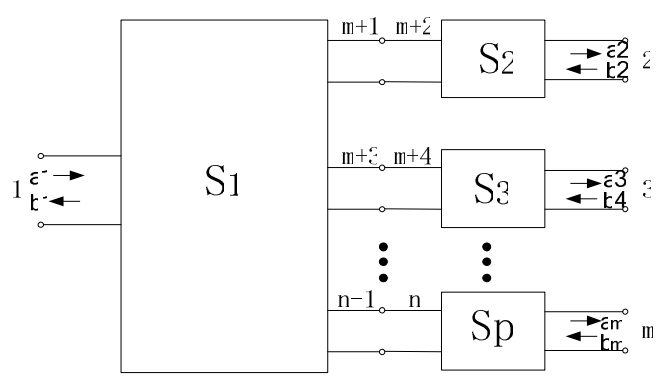

Figure 4 Multiple port network connection

The scattering matrix of each subnetwork is assumed as $S_{1}, S_{2}, \cdots S_{p}$ then

$$
b_{1}=S_{1} a_{1} ; b_{2}=S_{2} a_{1} ; \cdots ; b_{p}=S_{p} a_{p}
$$

All matrixes can be written out according to the subnetworks and partitioned as follows[4]

$$
\left[\begin{array}{c}
b_{1} \\
b_{2} \\
\vdots \\
b_{m} \\
\hdashline b_{m+1} \\
\vdots \\
b_{n}
\end{array}\right]=\left[\begin{array}{cccc:ccc}
S_{11} & S_{12} & \cdots & S_{1 m} & S_{1 m+1} & \cdots & S_{1 n} \\
S_{21} & S_{22} & \cdots & S_{2 m} & S_{2 m+1} & \cdots & S_{2 n} \\
\vdots & \vdots & \ddots & \vdots & \vdots & \ddots & \vdots \\
S_{m 1} & S_{m 2} & \cdots & S_{m m} & S_{m m+1} & \cdots & S_{m n} \\
\hdashline S_{m+11} & S_{m+22} & \cdots & S_{m+1 m} & S_{m+1 m+1} & \cdots & S_{m+1 n} \\
\vdots & \vdots & \ddots & \vdots & \vdots & \ddots & \vdots \\
S_{n 1} & S_{n 2} & \cdots & S_{n m} & S_{n m+1} & \cdots & S_{n n}
\end{array}\right]\left[\begin{array}{c}
a_{1} \\
a_{2} \\
\vdots \\
a_{m} \\
\hdashline a_{m+1} \\
\vdots \\
a_{n}
\end{array}\right]
$$

The partitioned matrix in the equation was used as $b_{I}, b_{I I}, A, B, C, D, a_{I}, a_{I I}$, then

$$
\left[\begin{array}{l}
b_{I} \\
b_{I I}
\end{array}\right]=\left[\begin{array}{ll}
A & B \\
C & D
\end{array}\right]\left[\begin{array}{l}
a_{I} \\
a_{I I}
\end{array}\right]
$$

It can be obtained according to the port connection condition as Figure 4 shows that:

$$
b_{I I}=\varepsilon a_{I}
$$

in which

$$
\varepsilon=\left[\begin{array}{lllllll}
0 & 1 & 0 & 0 & \cdots & 0 & 0 \\
1 & 0 & 0 & 0 & \cdots & 0 & 0 \\
0 & 0 & 0 & 1 & \cdots & 0 & 0 \\
0 & 0 & 1 & 0 & \cdots & 0 & 0 \\
\vdots & \vdots & \vdots & \vdots & \ddots & \vdots & \vdots \\
0 & 0 & 0 & 0 & \cdots & 0 & 0 \\
0 & 0 & 0 & 0 & \cdots & 1 & 0
\end{array}\right]
$$

Make simultaneous equation of equation (4) and (5) and solve

$$
b_{I}=\left[A+B(\varepsilon-D)^{-1} C\right] a_{I}=S a_{I}
$$

In the equation, $S=A+B(\varepsilon-D)^{-1} C$ is called the scattering matrix of m-port network after subnetwork connection.

The above-mentioned method, by which the combinational network scattering matrix after connection is reckoned according to the subnetwork scattering matrixes, may cause the partitioned matrix $\mathrm{D}$ of the complete matrix to be a matrix with quite big order number which makes quite heavy computation to obtain $(\varepsilon-D)^{-1}$. For complicated combinational network system, every two can be connected one by one, and the scattering matrix of the complicated network can be finally obtained after sequential computation. Such as the combination of star-point diplexer, as Figure 2 shows, the $\mathrm{S}$ matrixes of network which consists of the filter and transmission line of both sides can be obtained first, and the total S matrix can be obtained then.

Let the scattering matrix of the network which consists of filter and transmission of each side of the diplexer be $S_{A}, S_{B}$ and the scattering matrix of the branch connector be $S_{T}$. Then the scattering matrix of the diplexer will be obtained:

$$
\begin{aligned}
& \mathrm{S}_{11}=\mathrm{S}_{\mathrm{A} 11}+\frac{1}{\text { Det }} \mathrm{S}_{\mathrm{A} 12} \mathrm{~S}_{\mathrm{A} 21}\left(\begin{array}{l}
\mathrm{S}_{\mathrm{T} 11}-\mathrm{S}_{\mathrm{T} 11} \mathrm{~S}_{\mathrm{B} 22} \mathrm{~S}_{\mathrm{T} 22} \\
+\mathrm{S}_{\mathrm{T} 12} \mathrm{~S}_{\mathrm{T} 21} \mathrm{~S}_{\mathrm{B} 22}
\end{array}\right) \\
& \mathrm{S}_{12}=\frac{1}{\text { Det }} \mathrm{S}_{\mathrm{A} 12} \mathrm{~S}_{\mathrm{T} 12} \mathrm{~S}_{\mathrm{B} 21} \\
& \mathrm{~S}_{13}=\frac{1}{\operatorname{Det}}\left(\begin{array}{l}
\mathrm{S}_{\mathrm{A} 12} \mathrm{~S}_{\mathrm{T} 12} \mathrm{~S}_{\mathrm{B} 22} \mathrm{~S}_{\mathrm{T} 23} \\
-\mathrm{S}_{\mathrm{A} 12} \mathrm{~S}_{\mathrm{T} 13}\left(\mathrm{~S}_{\mathrm{B} 22} \mathrm{~S}_{\mathrm{T} 22}-1\right)
\end{array}\right)
\end{aligned}
$$




$$
\begin{aligned}
& \mathrm{S}_{21}=\frac{1}{\text { Det }} \mathrm{S}_{\mathrm{B} 12} \mathrm{~S}_{\mathrm{A} 21} \mathrm{~S}_{\mathrm{T} 21} \\
& \mathrm{~S}_{22}=\mathrm{S}_{\mathrm{B} 11}+\frac{1}{\text { Det }} \mathrm{S}_{\mathrm{B} 12} \mathrm{~S}_{\mathrm{B} 21}\left(\begin{array}{l}
\mathrm{S}_{\mathrm{T} 22}-\mathrm{S}_{\mathrm{T} 11} \mathrm{~S}_{\mathrm{A} 22} \mathrm{~S}_{\mathrm{T} 22} \\
+\mathrm{S}_{\mathrm{T} 12} \mathrm{~S}_{\mathrm{T} 21} \mathrm{~S}_{\mathrm{A} 22}
\end{array}\right) \\
& \mathrm{S}_{23}=\frac{1}{\text { Det }} \mathrm{S}_{\mathrm{B} 12} \mathrm{~S}_{\mathrm{T} 13} \mathrm{~S}_{\mathrm{T} 21} \mathrm{~S}_{\mathrm{A} 22} \\
& -\frac{1}{\text { Det }} \mathrm{S}_{\mathrm{B} 12} \mathrm{~S}_{\mathrm{T} 23}\left(\mathrm{~S}_{\mathrm{T} 11} \mathrm{~S}_{\mathrm{A} 22}-1\right) \\
& \mathrm{S}_{31}=-\frac{1}{\text { Det }} \mathrm{S}_{\mathrm{A} 21}\left(\mathrm{~S}_{\mathrm{T} 31}\left(\mathrm{~S}_{\mathrm{B} 22} \mathrm{~S}_{\mathrm{T} 22}-1\right)-\mathrm{S}_{\mathrm{T} 21} \mathrm{~S}_{\mathrm{B} 22} \mathrm{~S}_{\mathrm{T} 32}\right) \\
& \mathrm{S}_{32}=-\frac{1}{\text { Det }} \mathrm{S}_{\mathrm{B} 21}\left(\mathrm{~S}_{\mathrm{T} 32}\left(\mathrm{~S}_{\mathrm{T} 11} \mathrm{~S}_{\mathrm{A} 22}-1\right)-\mathrm{S}_{\mathrm{T} 12} \mathrm{~S}_{\mathrm{A} 22} \mathrm{~S}_{\mathrm{T} 31}\right) \\
& \mathrm{S}_{33}=-\frac{1}{\text { Det }} \mathrm{S}_{\mathrm{T} 23}\left(\begin{array}{l}
\mathrm{S}_{\mathrm{B} 22} \mathrm{~S}_{\mathrm{T} 32}\left(\mathrm{~S}_{\mathrm{T} 11} \mathrm{~S}_{\mathrm{A} 22}-1\right)- \\
\mathrm{S}_{\mathrm{T} 12} \mathrm{~S}_{\mathrm{A} 22} \mathrm{~S}_{\mathrm{B} 22} \mathrm{~S}_{\mathrm{T} 31}
\end{array}\right) \\
& -\frac{1}{\text { Det }} \mathrm{S}_{\mathrm{T} 13}\left(\mathrm{~S}_{\mathrm{A} 22} \mathrm{~S}_{31 \mathrm{~T}}\left(\mathrm{~S}_{\mathrm{B} 22} \mathrm{~S}_{\mathrm{T} 22}-1\right)-\mathrm{S}_{\mathrm{T} 21} \mathrm{~S}_{\mathrm{A} 22} \mathrm{~S}_{\mathrm{B} 22} \mathrm{~S}_{\mathrm{T} 32}\right) \\
& \text { Det }=S_{A 22} S_{B 22} S_{T 11} S_{T 22}-S_{A 22} S_{B 22} S_{T 12} S_{T 21} \\
& -S_{A 22} S_{T 11}-S_{B 22} S_{T 22}+1
\end{aligned}
$$

For the star-point triplexer or the constant impedance diplexer which has more complicated structure, according to the algorithm of the diplexer, the $\mathrm{S}$ matrix of the network which consists of filter and transmission line can be reckoned first, and then branch connectors can be connected, and finally the scattering matrix of the triplexer can be obtained after sequential computation.

\section{SOFTWARE}

Matlab is a mathematic computation software platform in which high performance numerical computing and graphical presentation are hang together. This paper has designed a CAD software for multiplexers by using Matlab which has the advantages of high-speed of computing, convenient usage, high visualization and so on. According to the SNP document of branch connectors and the filters, the software chooses the frequency sweep, step length and the connection methods, and obtain the scattering parameters of the multiplexer. It can also adjust the length of the transmission line , optimize to obtain the optimal solution and supply for assembly in projects.

Figure 5 gives the flow path of the software development. However, it must be noted that, for the SNP files of the filters and the branch connectors, the frequency range of the filter must be included in that of branch connectors and the user defined frequency sweep cannot be above the frequency range of the branch connectors, the linear interpolation of the original data should be based on the frequency sweep. When the scattering parameter of each device's unified frequency is constructed. Generally there are two kinds of complex interpolation: one is interpolation for real part and imaginary part respectively, and the other is interpolation for module value and argument respectively. Since the first will cause certain oscillation of the complex module value, the software has adopted the second interpolation method.

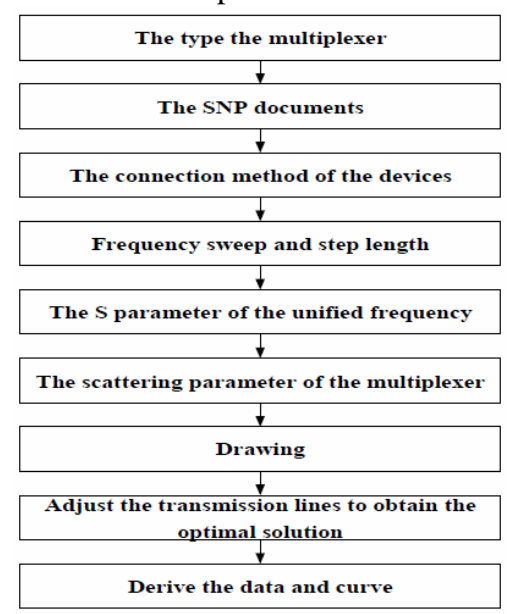

Figure 5 software development flow chart

\section{EXAMPLE}

\section{A. The star-point thiplexerB}

The branch connector of the star-point triplexer B consists of 2 T-joints and the connecting method of the device was given by Figure 6 . The passbands of the 3 filters are 480 488MHz, 486 504MHz, 512 520MHz. After importing the $s 2 p$ file of the filter and adjusting the length of transmission lines LA, LB, LC and LD, the S parameter curves of the starpoint riplexer can be obtained.

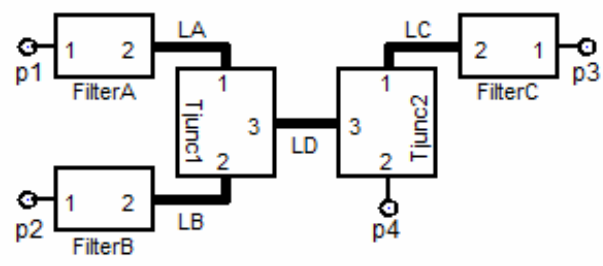

Figure 6 star-point triplexer B

And the same curve will be obtained as the software after the substitution of the numerical value of the transmission line into ADS simulation. The comparison of them is shown as in Figure $7 \&$ Figure 8.

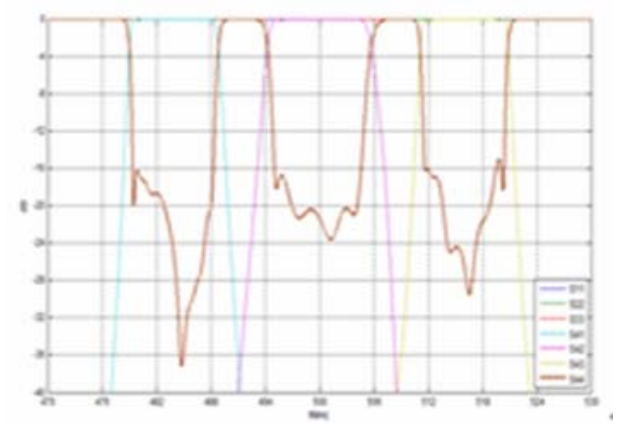

Figure 7 The comparative chart of the results of software and ADS simulation 


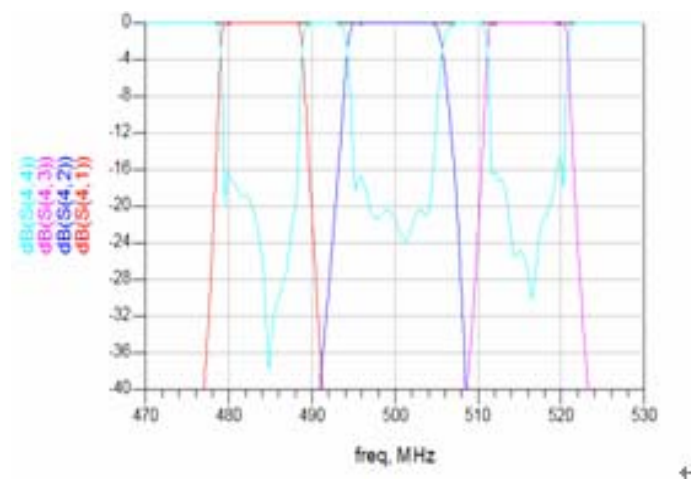

Figure 8 The S parameter curves obtained by ADS simulation

\section{B. The constant impedance diplexer}

The frequency range of the coupler is $3.4 \mathrm{GHz} \sim 4.2 \mathrm{GHZ}$, and the passband of the filter is $3.75 \mathrm{GHz} 3.85 \mathrm{GHZ}$. We choose the computer-aid design software of constant impedance diplexer to import the s2p files, and optimize the length of the transmission line. Figure 9 shows the S parameter curves of the diplexer.

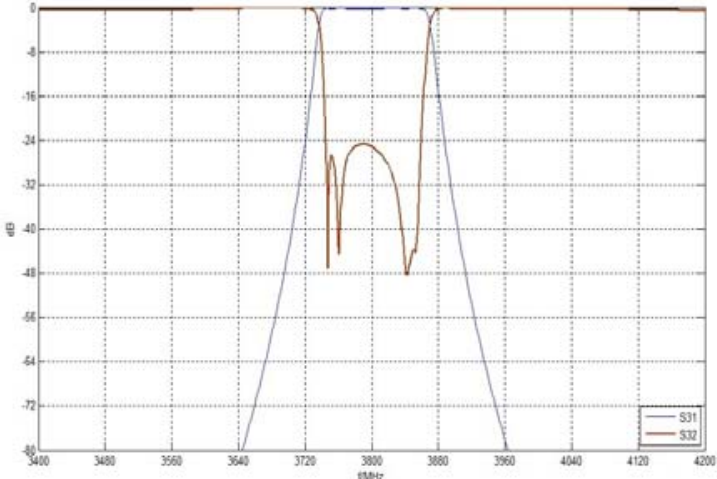

Figure 9 The S31 and S32 of the constant impedance diplexer

We verify the computer-aided design software of the constant impedance diplexer with ADS, and optimize the length of the transmission line obtaining the best effect. Figure 10 shows the simulation circuit in ADS.

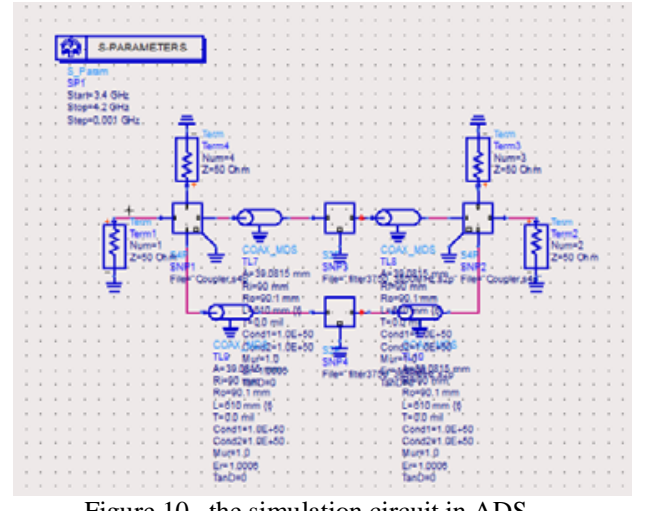

Figure 10 the simulation circuit in ADS.

Figure 11 and figure12 show the S31 parameter curve of the constant impedance diplexer in the polar coordinate system. As can be seen that the effect of the two are identical.

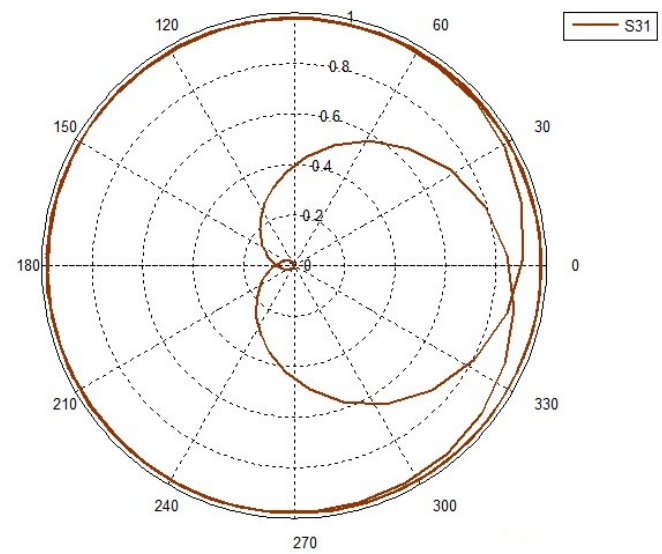

Figure 11 The S31 parameter curves in the polar coordinate system obtained by software simulation

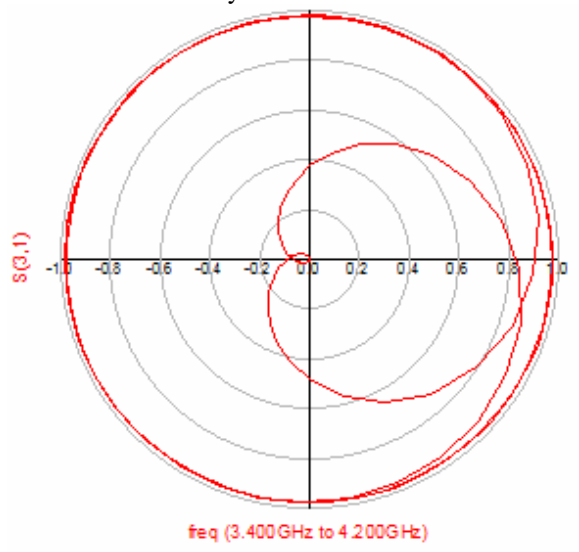

Figure 12 The S31 parameter curves in the polar coordinate system obtained by ADS simulation

\section{CONCLUSION}

This paper describes the working principle of the multiplexer and the method to obtain the network S matrix after connection by using the subnetwork S matrix. It has developed the multiplexer computer-aided design software of the starpoint diplexer, the star-point triplexer and constant impedance diplexer by using Matlab for eliminating residual susceptance and reducing the multiplexer design cycle, compared with the result of ADS simulation and found its advantage of high speed and accuracy.

\section{REFERENCES}

[1] Luo,YM.,\&Jv,JL(2011), Summary of multi-channel synthesizer ,Radio \& Television Information.

[2] Bai,SS.(2011), FM radio antenna synthesizer ,Cable Technology.

[3] Yan ,RQ.,\&Li,YH.(2004).Microwave technology base [M].Beijing: Beijing Institute of Tech-nology Press.

[4] Yang,SS.,\&Zhang,XJ. (2010)Linear Algebra, Beijing University of Posts and Telecommunica-tions Press.

[5] Shang,HC.(1996). Microwave network, Nanjing: Southeast University Press.

[6] Dong,L.(2008), Explain the use of Matlab, Beijing: Science Press. 